\title{
Amino Acid-Based Material for the Complementary Therapy of Decubitus Ulcers
}

\author{
Frederico Nogueira $^{1,2}$ and Isabel C. Gouveia ${ }^{2 *}$ \\ ${ }^{1}$ CICS-UBI - Health Sciences Research Centre, University of Beira Interior, Portugal \\ ${ }^{2}$ FibEnTech RED Unit Textile and Paper Materials, University of Beira Interior, Portugal
}

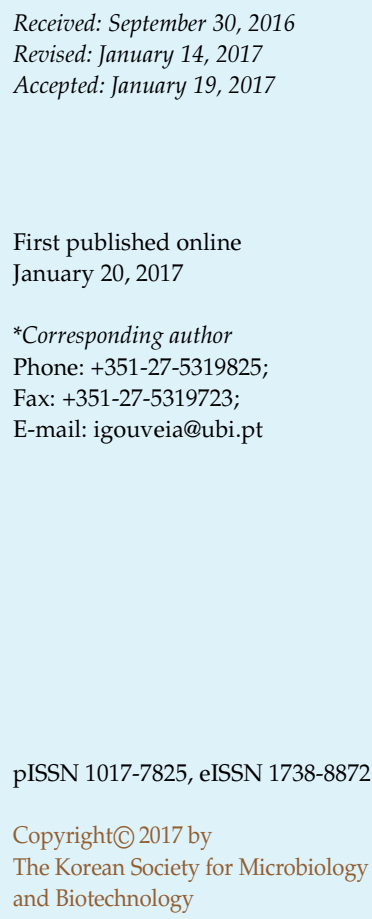

\begin{abstract}
Chronic wounds, pressure sores, lesions, and infections of microbial origin in bedridden, paralyzed, or malnutrition patients remain the object of study of many researchers. A variety of factors behind the development of these disorders are related to the patient's immune system, making it unable to respond effectively to the treatment of the wound. These factors can be properly controlled, giving particular importance to the ethiology and stage of the wound, as well as the time periods corresponding to the replacement of the dressings. The present research reports a novel foam/soft material, L-Cys-g-PCL, with an application for decubitus/pressure ulcers, especially for wounds with a difficult healing process due to infections and constant oxidation of the soft tissues. During this work, the interactions between S. aureus and L-Cys-g-PCL foam were studied under conditions that simulate decubitus ulcers; namely, $\mathrm{pH}$ and exudate. The effects of duration of grafting ( 1 or $8 \mathrm{~h})$ and $\mathrm{pH}(7.0$ and 8.9) on wettability, surface energy, swelling, and porosity were also evaluated. Results showed an effective microbicidal activity exhibiting an inhibition ratio of $99.73 \%$ against $S$. aureus. This new L-Cys-g-PCL soft material showed saftey to contact skin, ability to be shaped to fill in sunken holes (craters) - pressure ulcers stage III - and to act as a smart material responsive to $\mathrm{pH}$, which can be tailored to develop better swelling properties at alkaline $\mathrm{pH}$ where exudates are normally higher, so as to address exudate self-cleaning and prevention of desiccation.
\end{abstract}

Keywords: L-Cysteine, antimicrobial agents, chronic wounds, aminolysis, Staphylococcus aureus

\section{Introduction}

Decubitus ulcers (DUs), also called pressure ulcers or bedsores, are vascular disorders that are the result of prolonged pressure, friction, or shear over skin, especially at bony prominences $[1,2]$. DUs are more likely in the elderly, ill-fed, or individuals who stay immobile for a long while, which include the bedridden and paralyzed [2,3]. Frequent repositioning to scatter the weight on the surface of the skin and the use of special beds and cushions that reduce pressure through redistribution of load over a larger area of the equipment are essential parameters to prevent and control DUs [1]. Its prevalence has been increasing over the past decade, affecting about 7.7 million people worldwide [4]. Smoking, cold weather, hypertension, diabetes, hypercholesterolemia, and ischemic heart disease are contributors to the spread of pressure ulcers, with clinical long-term consequences, such as wounds that cannot heal within 3-4 months [5] - chronic wounds. Furthermore, patients can develop ischemia in the areas of inefficient blood circulation, a condition in which blood products, such as circulating cells, nutrients, and oxygen are delivered to the tissue below a fair amount. Hypoxia is, in this way, a direct consequence of ischemia [6]. The healing tissue demands no homeostatic but higher energy, and hence, a more oxygenated microenvironment, to promote adequate regenerative complexes that work for tissue repair [7]. Oxygen has a major role in the synthesis of collagen, induction of blood-vessel growth, regeneration of the epithelium, and fighting off infections. Although acute and mild hypoxia are described as angiogenic in a tumor context [8], extreme chronic wound hypoxia is not 
compatible with tissue repair [7]. Furthermore, tissues in a near-anoxic state can progress towards necrosis, which may spread to surrounding tissue, leaving the body highly vulnerable to microbial infections [9]. After a trauma, immune system triggers vasodilation and consequently vessel permeability with wound exudate as a final outcome. This fluid presents cells, key factors for healing, and a great ability to acquire microorganisms [10]. In chronic wounds, persistent inflammation and microbial infections can become established. Chronic exudates have a different composition and appearance than acute ones, and have deleterious effects on growth factors and peri-wound skin [11]. In chronic inflammation, the expression of mediators of inflammation is out of control; for instance, heparinbinding protein, with enhanced vascular permeability and a consequent abnormal production of exudate [12, 13]. Bacteria that colonize chronic wounds, most of them normal skin microbiota, also contribute to histamine release [14]. Moreover, resistant bacteria can also become established and have enough time to proliferate [15], which may kill the patient and spread to the community.

The success of treatment of a chronic wound involves elimination of bacterial colonization, proper oxic conditions provided by wound-dressing structure, temperature above $33^{\circ} \mathrm{C}$, growth factors, wound hydration (optimum moisture balance), exudate absorption (hydrophilicity), necrosis tissue avoidance, and prevention of maceration of surrounding tissue [16-18].

DUs are a huge reservoir of $S$. aureus, which can also be present in human skin without causing any infection. It is enclosed in a capsule that prevents engulfment by PMNs, along with a slime layer made up of biopolymers that can develop attachments to various human receptors as well as external biomedical devices. Additionally, 11 capsular serotypes are identified. S. aureus produces a range of toxins that operate as virulence factors: 5 cytolytic that detroy the cellular membrane, 2 exfoliative, 20 enterotoxins, and 1 TSST [19]. S. aureus causes clinical disease when it releases different toxins, carries out colonization, and brings about the necrosis of tissues. Although manifestation of the disease relies largely on the action of toxins, in a few situations the cause lies in the S. aureus growth that makes over abscess and tissue necrosis. Likewise, it provides resistance to the commonly used (and essentially every) antibiotics [20], a serious cause for concern in DUs [21]. The good progress in antimicrobial wound dressings provides a reliable solution to the adjuvant antimicrobial therapy for chronic disease.

The polyester polycaprolactone (PCL) is a non-toxic hydrophobic synthetic tissue-compatible polymer, being one of the five degradable polymers that the Food and Drug Administration officially approved to meet the needs of healthcare [22]. Pressure ulcers stage III are very difficult to treat and may require years to heal [1]. The polyester PCL has two highly characteristic properties especially suitable for pressure ulcers stage III: (i) its slow the biodegradability rate, which renders PCL an attractive drug delivery device for more than a year (2-3 years) [23]; (ii) and its low tensile strength, yet very high elongation at breakage turn PCL fascinatingly elastic. These are the desirable characteristic features for the regeneration of skin and vascular tissues [24]. On the other hand, other materials, such as hydrogels, bring lower stability: they require environmental control of humidity, nutrients, and microorganisms, which seem unrealistic to control under in vivo conditions; they are susceptible to enzymatic and microbial degradation, have low mechanical resistance, and last no more than a few weeks [22]. As with all biodegradable polymers, PCL is more or less degraded according to its own physicochemical composition; namely, molecular weight, degree of crystallinity, and geometry (form, dimension, porosity/void fraction, and ratio of surface to volume) [25].

This work describes the innovative use of L-Cysteine (LCys) that is part of diverse food sources. This $\alpha$-amino acid is thiol-ended, which encompasses a wide range of antimicrobial activity [26], acting as a disrupter of metabolism and the membrane [27]. L-Cys aims particularly at the microbial membrane and compromises its enzymatic and the metabolic machinery. Its capability for conjugating with reactive chemical species and trace elements [28] adds the ability to counteract the damaging effects of oxidation in human tissues.

The present work takes account of the development of a process that granted the PCL foam with the covalent linkage of L-Cys (L-Cys-g-PCL), which also converted it into a hydrophilic material for exudate absorption purposes. Herein, we present new findings claiming the development of a new foam-shaped wound dressing with an amino acid for DUs. The development of biomaterials that provide comfort, antioxidant, antimicrobial, and non-cytotoxic properties, has become a priority of research.

\section{Materials and Methods}

\section{Reagents}

Polycaprolactone and 1,4-dioxane as solvent (>99\%) were from Sigma (USA). 
Staphylococcus aureus, strain ATCC 6538, was cultivated in solid nutrient agar and liquid nutrient broth (Panreac, Spain), with vigorously stirring.

\section{Foam Preparation}

A solution of PCL, $M_{n} 45,000,3 \%(w / v)$ in dioxane solvent, was prepared in a fume hood, stirring $2 \mathrm{~h}$ until complete dissolution. The solution was then placed in a freezer at $-80^{\circ} \mathrm{C}$ overnight, and subsequently freeze-dried for $48 \mathrm{~h}$. Foam cross-sections were made and stored in a desiccator until use.

Foam samples were covalently functionalized via aminolysis with amine compounds present in 5\% (w/v) L-Cysteine (L-Cys) solution (Sigma-Aldrich, Germany), for 1 and $8 \mathrm{~h}$, under $\mathrm{pH} 6.5$ at $40^{\circ} \mathrm{C}$.

\section{Methods}

The foam specimen was evaluated by surface/core analyzing procedures and further investigated for antimicrobial activity and swelling profiles to estimate its behavior and potential as a wound dressing for DUs.

\section{Contact Angle}

OCAH-200 (Dataphysics, USA) was used to record contact angles, using a high-speed video hardware with a resolving power of $752 \times 484$.

\section{Free Energy of Adhesion}

The surface tension of samples was ascertained using the sessile drop method at room temperature, using one non-polar component (diiodomethane) and two polar solvents (water and ethylene glycol), whichever surface tension components are known:

$$
(1+\cos \theta) \gamma 1^{\mathrm{TOT}}=2\left(\sqrt{\gamma \mathrm{s}^{\mathrm{LW}}} \gamma 1^{\mathrm{LW}}+\sqrt{\gamma \mathrm{s}^{+}} \gamma 1^{-}+\sqrt{\gamma \mathrm{s}^{-}} \gamma 1^{+}\right)
$$

The symbol $\theta$ means the contact angle; also $\gamma^{\mathrm{TOT}}=\gamma^{\mathrm{LW}}+\gamma^{\mathrm{AB}}$. The parameter $\gamma^{\mathrm{LW}}$ corresponds to the Lifshitz-van der Waals element of the surface free energy; $\gamma^{+}$is the electron acceptor, and $\gamma^{-}$is the electron donor of the Lewis acid-base $\gamma^{\mathrm{AB}}$, knowing that $\gamma^{\mathrm{AB}}=2$ $\sqrt{\gamma^{+}} \gamma^{-}[29]$.

The total interaction energy $\Delta G_{\text {Adhesion }}{ }^{\text {TOT }}$ was predicted from the interactions between $S$. aureus ${ }^{[2]}$ and foam ${ }^{[1]}$ :

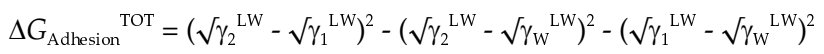

$$
\begin{aligned}
& +2\left[{\sqrt{\gamma_{W}}}^{+}\left({\sqrt{\gamma_{2}}}^{-}+\sqrt{\gamma_{1}-}-\sqrt{\gamma_{\mathrm{W}}}\right)+{\sqrt{\gamma_{\mathrm{W}}}}^{-}\left({\sqrt{\gamma_{2}}}^{+}+{\sqrt{\gamma_{1}}}^{+}-\sqrt{\gamma_{\mathrm{W}}}{ }^{+}\right)-\sqrt{\gamma_{2}{ }^{-} \gamma_{1}{ }^{+}-}\right. \\
& \sqrt{\left.\gamma_{2}^{+} \gamma_{1}^{-}\right]}
\end{aligned}
$$

If $\Delta G_{\text {Adhesion }}{ }^{\text {ТОт }}<0$, the expected adhesion of $S$. aureus is considered favorable. Conversely, the expected adhesion is not considered favorable if $\Delta G_{\text {Adhesion }}{ }^{\text {TOT }}>0$ [29].

\section{Energy Dispersive X-ray Spectroscopy (EDS)}

An EDS interconnected to a scanning electron microscope (HITACHI S 2700, Japan) was used to quantify the sulfur presence on the samples.

\section{Ellman's Assay (Datacolor)}

Ellman's reagent, (5,5'-dithio-bis-(2-nitrobenzoic acid) (Sigma, USA) turns yellow immediately upon conjugating with free thiol groups, where it forms a dissulfidric compound called 2-nitro-5thiobenzoic acid. This test was conducted to measure thiolates of L-Cys-modified PCL samples, allowing their quantification through determined $K / S$ values. Briefly, samples were immersed in a bath ratio of $1 / 60$ of Ellman's reagent at $40^{\circ} \mathrm{C}$ for $45 \mathrm{~min}$, under $15 \mathrm{rpm}$ stirring, using a Mathis-BFA12. Color measurement was performed using the spectrophotometer Spectraflash SF300 (Datacolor, Switzerland). Reflectance $(R)$ was measured at $412 \mathrm{~nm}$ wavelength of the visible light zone and it was intimately linked to concentration with the Kubelka-Munck formula:

$$
K / S=(1-R)^{\wedge} 2 / 2 R
$$

$R$ represents reflectance, $K$ constitutes the absorption coefficient, and $S$ stands for the diffusion coeficient, because

$$
K / S=\alpha C
$$

where $C$ is the dye concentration in the fiber and $\alpha$ a constant.

\section{Structural Analysis}

The chemical contents of PCL foam, as well as foams grafted with L-Cys for 1 and $8 \mathrm{~h}$, were determined with a Thermo-Nicolet is10 Fourier transform infrared spectrophotometer (FT-IR). The polarized spectra were scanned 64 times, with a spatial frequency resolving power of $4 \mathrm{~cm}^{-1}$.

\section{X-Ray Diffraction (XRD) Analysis}

The crystal structure of the samples was assessed by XRD, after being crushed to powders. Scans were performed between $2 \theta$ values of $5^{\circ}$ and $90^{\circ}$.

\section{Differential Scanning Calorimetry}

The crystallinity of the $1 \mathrm{~h}$ L-Cys-g-PCL was further investigated with differential scanning calorimetry (DSC) (DSC 204 Phoenix (Netzsch, Germany)) with samples completely filled in small aluminum containers. Nonisothermal scans were carried out from $30^{\circ} \mathrm{C}$ to $300^{\circ} \mathrm{C}$ at a heating rate of $2^{\circ} \mathrm{C}$ per minute, with a nitrogenreplacing atmosphere. The melting point $\left(T_{m}\right)$ and enthalpy of fusion $\left(\Delta H_{\mathrm{m}}\right)$ were obtained.

\section{Swelling Ratio}

Pre-weighted foam samples (W1) were immersed in a solution ratio of $0.1 \mathrm{~g}$ sample weight: $50 \mathrm{ml}$ of $\mathrm{ddH}_{2} \mathrm{O}$ adjusted to $\mathrm{pH} 7$ and 8.9 , under $25^{\circ} \mathrm{C}$. At 0,2 , and $24 \mathrm{~h}$, samples were taken from the solution, wiped, and weighed (W2). The amount of swelling was determined according to Eq. (4).

$$
\text { Swelling ratio }(\%)=(W 2-W 1) \times 100 / W 1
$$

\section{Porosity and Density}

The density was determined based on Archimedean Principle, 
by weighing the samples at the room environment and immersed in a liquid $\left(\mathrm{ddH}_{2} \mathrm{O}\right)$ at $\mathrm{pH} 7$ and 8.9. Providing that the principle of density determination is $\rho=m(\mathrm{~g}) / V\left(\mathrm{~cm}^{3}\right)$, the sample density is

$$
\rho_{2}=(A /(B-A)) \times \rho_{1}
$$

where $A$ represents the weight of the sample in the room environment, $B$ stands for the weight of the submerged sample, and $\rho_{1}$ is the density of the test liquid (water) at the temperature of $37^{\circ} \mathrm{C}$. The density measurements provided information about the porosity, where

$$
\% \text { porosity }=[1-(\text { foam density } / \text { bulk density })] \times 100
$$

The bulk density of PCL is $1.15 \mathrm{~g} / \mathrm{cm}^{3}$ (Sigma Aldrich).

\section{Anti-S. aureus Activity}

An assay was carried out to determine the bacteria viability reduction with the application of the L-Cys-functionalized foam samples, according to E 2180-01 Standard Test Method for Determining the Activity of Incorporated Antimicrobial Agent(s) in Polymeric or Hydrophobic Materials. The gram-positive bacterial strain Staphylococcus aureus (ATCC 6538) was chosen as it is a common pathogen present in nosocomial infections, especially in bedsore ulcers. Briefly, inoculum of $S$. aureus culture was prepared with $\mathrm{NaCl}$ and agar-agar in order to form a agar slurry with 1-5 $\times$ $10^{6}$ bacteria/ml, which was poured $(1 \mathrm{ml})$ onto $3 \times 3 \mathrm{~cm}$ square samples, with subsequent incubation for $24 \mathrm{~h}$. The antimicrobial efficiency was quantitatively evaluated, where the percentage of bacterial reduction (\%R) was calculated at 0 and $24 \mathrm{~h}$ using:

$$
\% \text { Reduction }=\frac{C-A}{C} \times 100
$$

where $C$ is the $\mathrm{CFU}$ (colony forming units) on the control sample and $A$ is the CFU on the functionalized foam (L-Cys-g-PCL).

In order to ascertain whether the samples have a bacteriostatic or bactericidal effect, the following equations were used [30]:

$$
\begin{aligned}
& \text { Bacteriostatic activity }=M_{\mathrm{b}}-M_{\mathrm{c}} \\
& \text { Bactericidal activity }=M_{\mathrm{a}}-M_{\mathrm{c}}
\end{aligned}
$$

where $M_{\mathrm{a}}$ is $\log _{10}$ of the average of three replicas of $T_{0 \mathrm{~h}}$ control samples, $M_{\mathrm{b}}$ corresponds to $\log _{10}$ of the average of three replicas at $T_{24 h}$ control samples, and $M_{c}$ relates to $\log _{10}$ of the average of three replicas at $T_{24 \mathrm{~h}}$ treated samples.

\section{Scanning Electron Microscopy (SEM)}

A fixation solution of $1.5 \%$ gluteraldehyde was prepared and added to the adsorbed bacteria on samples, which were then incubated overnight at $4^{\circ} \mathrm{C}$. Afterwards, the samples were dehydrated at percentage values starting from 50\% until $99 \%$, with ethanol plus water, and then dried (K850 Emitech, UK). After covering the samples with a thin coating of gold, they were examined under a scanning electron microscope (Hitachi S2700, Japan) at a high voltage of $20 \mathrm{kV}$.
The purpose of SEM analysis was to quantify and compare S. aureus attachment after $24 \mathrm{~h}$, with an initial innoculum of $10^{7}$ S. aureus $/ \mathrm{ml}$, as well as the size of the pores. Pore sizes were measured using the equation: $d=l \times h$ [31], where $l$ means the average maximum pore length and $h$ is the average minimum pore length.

\section{Cytotoxicity Assay}

The testing of cytotoxicity of L-Cys-g-PCL on mouse fibroblast cell line BALB/c 3T3 was done based on an adaptation of E DIN EN ISO 10993-5. Two different treatments were performed: (i) sterilized L-Cys-g-PCL discs $\left(0.56 \mathrm{~cm}^{2}\right)$ were poured floating at the center of each well; and (ii) perspiration extracts of $4.4 \%, 6.6 \%$, $9.9 \%, 14.8 \%, 22.2 \%$, and $33.3 \%$ were added. The samples were incubated for $24 \mathrm{~h}$ and at $37^{\circ} \mathrm{C}$, under $5 \% \mathrm{CO}_{2}$. The MTS test of cell viability was done, including solvent and negative controls.

\section{Statistical Analysis}

The significant values $(p<0.05)$ were calculated according to the multiple comparisons between results, with one-way ANOVA and post-hoc Tukey tests (SPSS 21.0).

\section{Results}

\section{Contact Angle}

The wettability of PCL foam, together with PCL grafted foams at 1 and $8 \mathrm{~h}$ (L-Cys-g-PCL), was calculated according to the angle between the samples surface and the water drop. These angles were recorded at $10 \mathrm{sec}$, because they proved stability and allowed us to observe a native hydrophobic PCL foam surface; $109.9^{\circ} \pm 5.0^{\circ}$. Interestingly, L-Cys-g-PCL for 1 and $8 \mathrm{~h}$ were found to be significantly different from the latter, turning to hydrophilic surfaces of $86.5^{\circ} \pm 10.0^{\circ}$ and $58.1^{\circ} \pm 16.1^{\circ}$, respectively.

\section{Free Energy of Adhesion}

The interaction potential for bringing $S$. aureus closer to the samples was determined by a thermodynamic approach of the free energy [29]. It was determined that the ability of the $S$. aureus strain studied was unfavorable to bind to the control, which consisted of PCL $\left(\Delta G_{\text {Adhesion }}{ }^{\text {TOT }}=7.17\right)$. When the same bacterium was exposed to $1 \mathrm{~h}$ L-Cys-g-PCL $\left(\Delta G_{\text {Adhesion }}^{\text {TOT }}=19.14\right)$ and $8 \mathrm{~h} \mathrm{L-Cys-g-PCL}\left(\Delta G_{\text {Adhesion }}{ }^{\text {TOT }}=\right.$ 19.99), the $\Delta G_{\text {Adhesion }}{ }^{\text {TOT }}$ increased, meaning that the adhesion capacity of $S$. aureus to grafted PCL decreased with the introduced modifications.

\section{Energy Dispersive X-Ray Spectroscopy}

The amount of sulfur groups present in the grafted samples was calculated with EDS analysis, which is used as 
Table 1. Color strength $(K / S)$ correlated to functionalization by L-Cys of controls, $1 \mathrm{~h}$ L-Cys-g-PCL, and $8 \mathrm{~h}$ L-Cys-g-PCL.

\begin{tabular}{cc}
\hline Foam samples & $K / S(400 \mathrm{~nm})$ \\
\hline Blank & $0.03 \pm 0.02$ \\
1 h L-Cys-g-PCL & $0.26 \pm 0.09$ \\
8 h L-Cys-g-PCL & $0,16 \pm 0.06$ \\
\hline
\end{tabular}

an analytical technique for elemental analysis and chemical characterization. The graft presence varied with the modification time. The absence of sulfur in the PCL native structure helped us to determine indirectly the success of the grafting. The $1 \mathrm{~h}$ of grafting was bound to bind an increased load of L-Cys (i.e., 1.64\% wt. (percentage by weight)). On the other hand, $8 \mathrm{~h}$ of grafting retained only $0.32 \%$ wt. In the absence of grafting (controls), no sulfur traces were detected.

\section{Colorimetric Assay (DataColor)}

Additionally, to confirm the correlation of sulfur content to thiolates, colorimetric assay with Ellman's reagent was performed. Results of the color strength based on the amount of functionalized L-Cys are presented in Table 1.

\section{Structural Analysis (FT-IR)}

The native PCL spectrum is presented in Fig. 1a. This

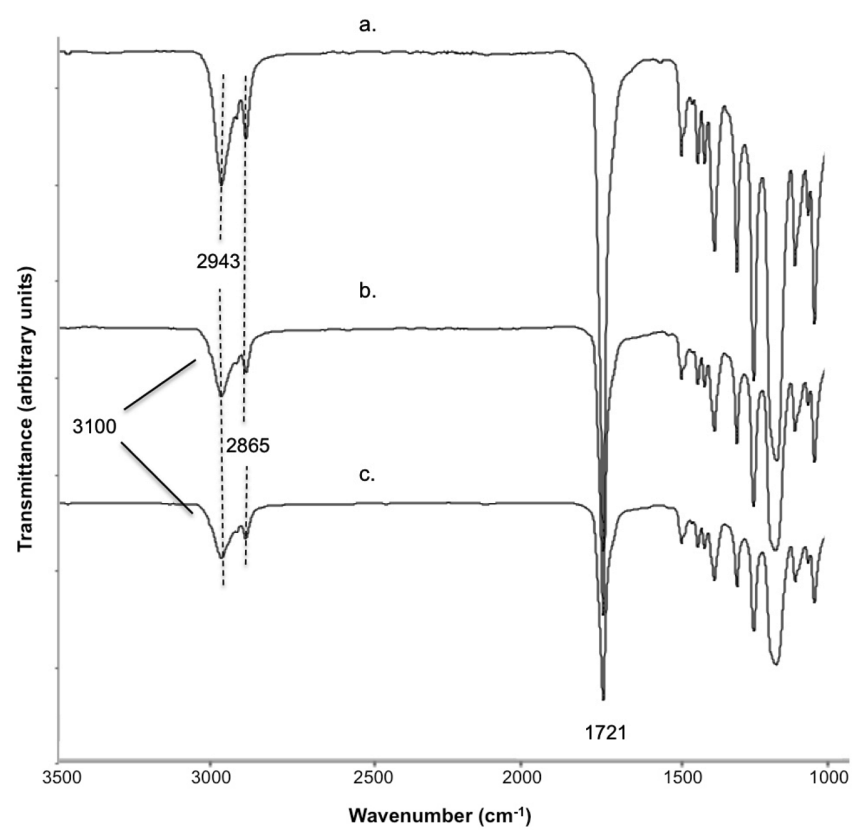

Fig. 1. IR spectra of unmodified polycaprolactone (PCL) foam (a), $1 \mathrm{~h}$ L-Cys-g-PCL (b), and $8 \mathrm{~h}$ L-Cys-g-PCL (c) both with $5 \%$ (w/V) L-Cys. shows the expected characteristic peaks $\mathrm{C}-\mathrm{H}$, associated with $\mathrm{CH}_{2}$ stretching vibrations at $2,943 \mathrm{~cm}^{-1}$ and $2,865 \mathrm{~cm}^{-1}$ and with carbonyl adsorption at $1,721 \mathrm{~cm}^{-1}$ [32].

Structural differences accomplished on PCL after its covalent modification, at two times of grafting ( 1 and $8 \mathrm{~h}$ ), were also estimated. Infrared spectra confirmed the PCL chain grafted with L-Cys, marked by the rise of the peak owing to its hydroxyl functional group, around $3,100 \mathrm{~cm}^{-1}$, as shown in Fig. 1b and 1c.

\section{X-Ray Diffraction Analysis}

A careful look at the XRD spectra allowed us to see the phase composition and crystal structure of the PCL foam control (Fig. 2a), $1 \mathrm{~h}$ L-Cys-g-PCL (Fig. 2b), and $8 \mathrm{~h}$ L-Cysg-PCL (Fig. 2c). The PCL foam and grafted ones showed their main peaks typical of an orthorhombic crystalline polyethylene-like unit cell at $21.5^{\circ}$ and $23.5^{\circ}$, corresponding to (110) and (200) reflections, respectively [33]. Although the peak relative intensity values for $2 \theta$ of $21.5^{\circ}$ and $23.5^{\circ}$ were similar for the control (a) and $8 \mathrm{~h} \mathrm{L-Cys-g-PCL} \mathrm{(c),} \mathrm{the}$ intense diffraction peak at $21.5^{\circ}$ for $1 \mathrm{~h} \mathrm{L-Cys-g-PCL} \mathrm{(b)}$ appeared with no shift, albeit with a higher intensity than those of the latter, indicating the same crystal form with a high-ordered structure. This evidence could suggest a rather more crystalline structure for $1 \mathrm{~h} \mathrm{~L}-\mathrm{Cys}-\mathrm{g}-\mathrm{PCL}$, when comparing with the control and $8 \mathrm{~h}$ L-Cys-g-PCL.

\section{Differential Scanning Calorimetry}

Based on XRD evidence, the effect of $1 \mathrm{~h}$ L-Cys-g-PCL and its thermal properties were investigated. Melting temperatures $\left(T_{\mathrm{m}}\right)$ for the control, $1 \mathrm{~h} \mathrm{~L}-\mathrm{Cys}-\mathrm{g}-\mathrm{PCL}$, and $\mathrm{L}$ Cys were obtained from the heating scan, and were $63^{\circ} \mathrm{C}$, $62^{\circ} \mathrm{C}$, and $182^{\circ} \mathrm{C}$, respectively (Fig. 3). These values suggest that the covalent introduction of $5 \%(\mathrm{w} / \mathrm{v})$ L-Cys graft affected the PCL crystallinity degree. Covalent bonds do not break on melting or dissolving. Moreover, molecules are held together by weak intermolecular forces in covalent solids. When a solid melts, the forces that are overcome are the weaker ones, keeping the covalent bonding intact. This may explain why $1 \mathrm{~h}$ L-Cys-g-PCL decreased its melting point. Furthermore, its decreased enthalpy of fusion $(0.58$ $\mathrm{mW} / \mathrm{mg}$ ) might be due to first the latter reason, and second the aminolysis reaction, where the PCL chain is split in two parts. The enthalpy of fusion for the control was $0.67 \mathrm{~mW} / \mathrm{mg}$.

\section{Swelling Ratio}

The swelling ratio (\%) of the studied samples was determined in $\mathrm{pH} 7$ (Fig. 4A) and pH 8.9 (Fig. 4B), at a ratio 

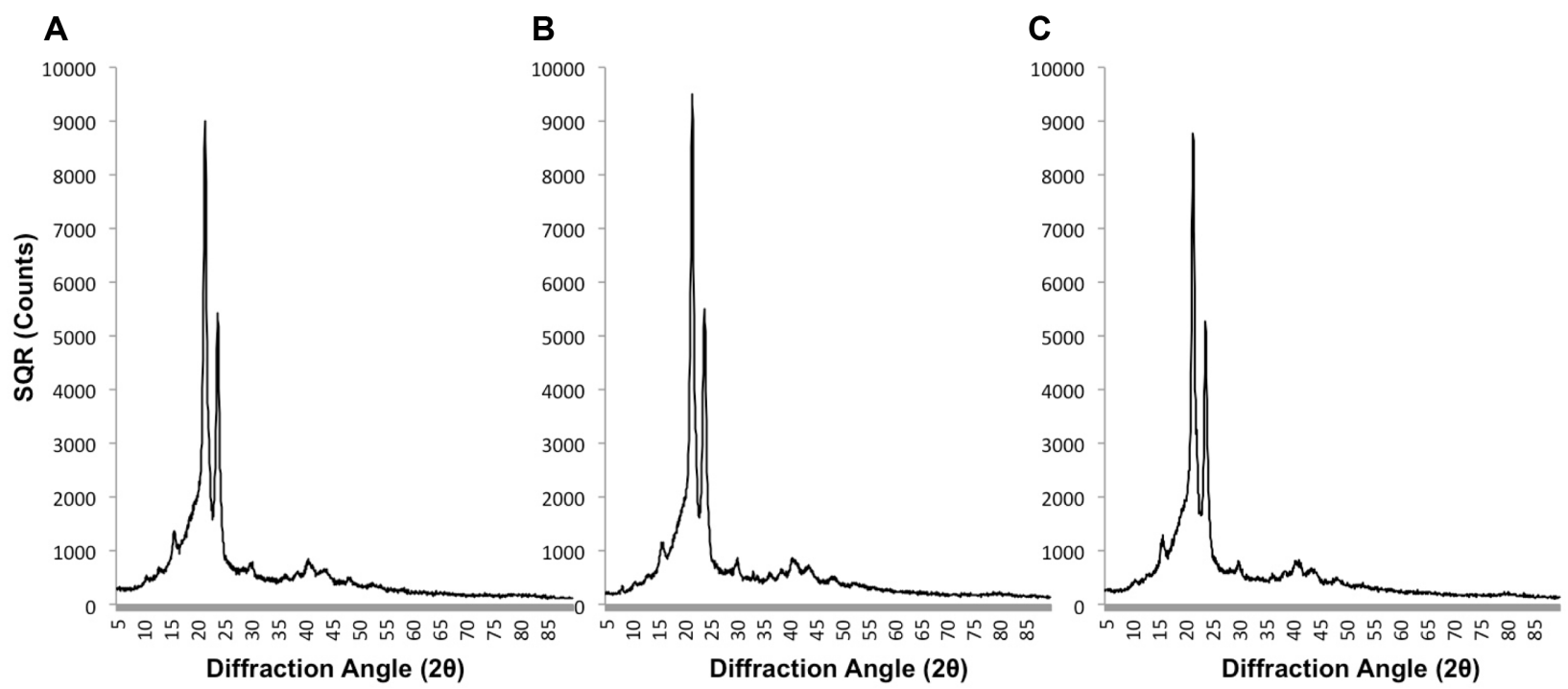

Fig. 2. XRD spectra of sample discs for unmodified polycaprolactone (PCL) (A), $1 \mathrm{~h}$ L-Cys-g-PCL (B), and $8 \mathrm{~h} \mathrm{L-Cys-g-PCL} \mathrm{(C),}$ both with $5 \%(\mathrm{w} / \mathrm{v})$ L-Cys.

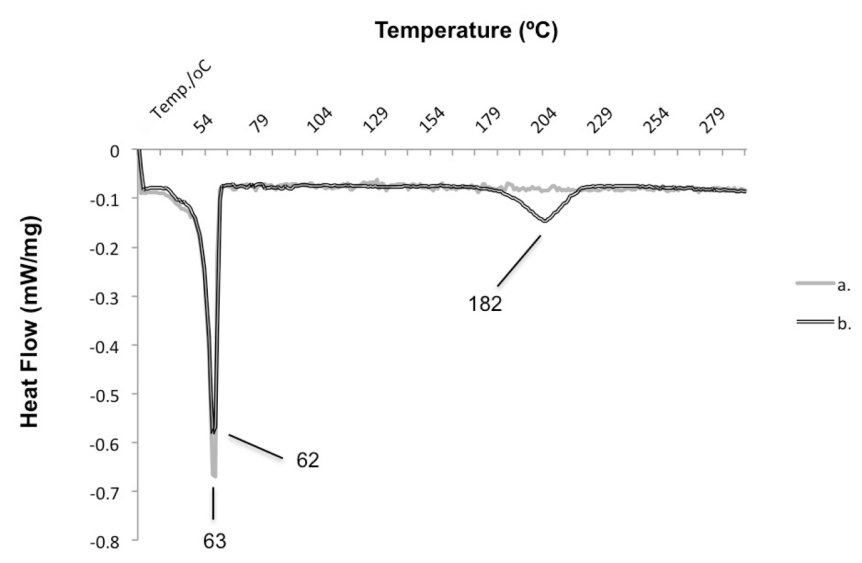

Fig. 3. Differential scanning calorimetry spectra of control polycaprolactone (PCL) foam (a) and the selected $1 \mathrm{~h}$ L-Cys-gPCL (b).

solution of $0.1 \mathrm{~g} / 50 \mathrm{ml}, 37^{\circ} \mathrm{C}$, at 0,2 , and $24 \mathrm{~h}$. Calculations were according to Eq. (4). All three samples, PCL foam (Fig. 4a), $1 \mathrm{~h}$ L-Cys-g-PCL (Fig. 4b), and $8 \mathrm{~h}$ L-Cys-g-PCL (Fig. 4c), showed time dependence of water absorbency. Furthermore, the absorbency was always the highest for $1 \mathrm{~h}$ L-Cys-g-PCL (Fig. 4b), and the lowest for PCL foam (Fig. 4a), except at pH 8.9 where 8 h L-Cys-g-PCL (Fig. 4c) after $24 \mathrm{~h}$ of incubation in solution was even lower. At $\mathrm{pH}$ 8.9 , the absorbency was incredibly high for $1 \mathrm{~h}$ L-Cys-gPCL (Fig. 4b), reaching swelling ratios of $382 \%$ after $2 \mathrm{~h}$ of incubation in solution, and $431 \%$ after $24 \mathrm{~h}$ of incubation in solution.
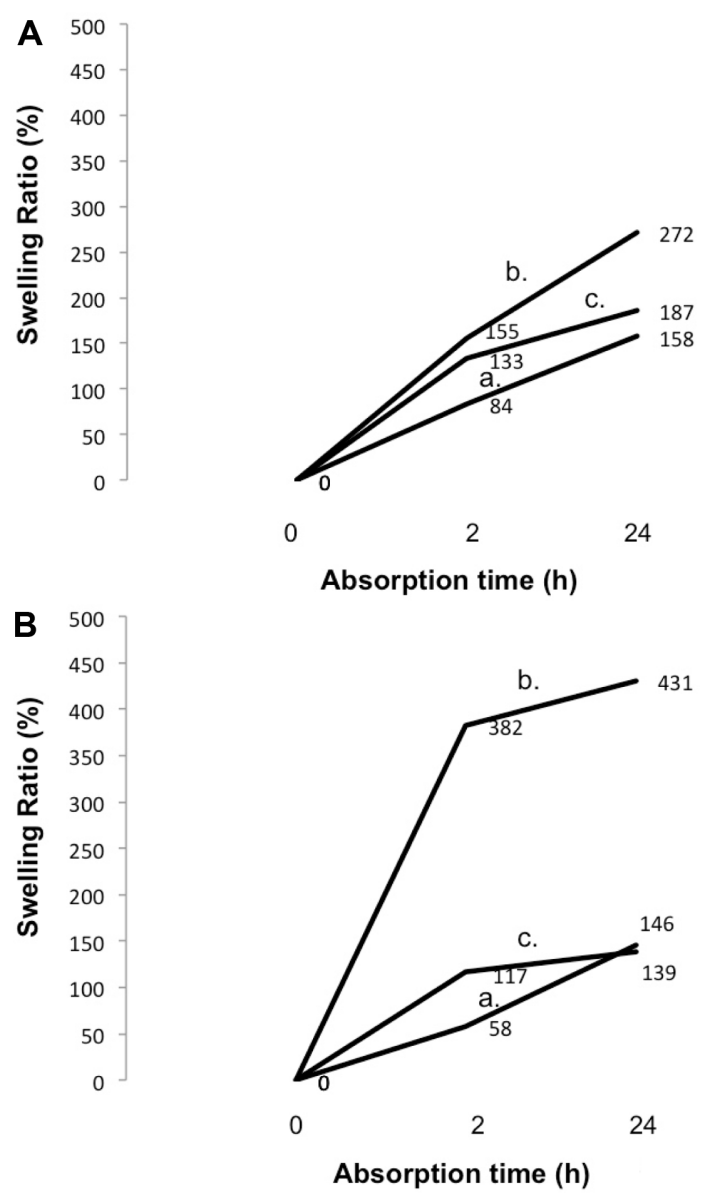

Fig. 4. Swelling (\%) of samples of polycaprolactone (PCL) (a), $1 \mathrm{~h} \mathrm{L-Cys-g-PCL} \mathrm{(b),} \mathrm{and} 8 \mathrm{~h} \mathrm{L-Cys-g-PCL} \mathrm{(c),} \mathrm{before} \mathrm{and} \mathrm{after}$ incubation in buffers at $\mathrm{pH} 7(\mathbf{A})$ and $8.9(\mathbf{B})$. 
Table 2. Densities and porosities of samples after $24 \mathrm{~h}$ of incubation under $\mathrm{pH} 7$ and 8.9 .

\begin{tabular}{ccccc}
\hline \multirow{2}{*}{$\begin{array}{c}\text { Foam samples } \\
(24 \mathrm{~h})\end{array}$} & \multicolumn{2}{c}{ Density $\left(\mathrm{g} / \mathrm{cm}^{3}\right)$} & \multicolumn{2}{c}{ Porosity $(\%)$} \\
\cline { 2 - 5 } & $\mathrm{pH}$ & $\mathrm{pH} \mathrm{8.9}$ & $\mathrm{pH} \mathrm{7}$ & $\mathrm{pH} \mathrm{8.9}$ \\
\hline Blank & 0.63 & 0.68 & 44.87 & 40.51 \\
1 h L-Cys-g-PCL & 0.37 & 0.23 & 68.01 & 79.82 \\
8 h L-Cys-g-PCL & 0.54 & 0.72 & 53.40 & 37.30 \\
\hline
\end{tabular}

\section{Porosity and Density}

The foams were produced with a porous structure. Their density and porosity were assessed after $24 \mathrm{~h}$, and are shown in Table 2. For both $\mathrm{pH}^{\prime}$, the porosity was always the highest for $1 \mathrm{~h} \mathrm{~L}-\mathrm{Cys}-\mathrm{g}-\mathrm{PCL}$, and the lowest for control PCL foam, except at $\mathrm{pH} 8.9$ where $8 \mathrm{~h}$ L-Cys-g-PCL was inferior. At $\mathrm{pH} 8.9$, porosity was very high for $1 \mathrm{~h}$ L-Cys-gPCL, averaging $79.82 \%$. This might be explained by the ionized L-Cys -OH and $-\mathrm{SH}$ groups at $\mathrm{pH} 8.9$, which repel each other.

\section{Anti-S. aureus Assays - Quantitative Method (Suspension)}

This assay evaluated the $S$. aureus viability reduction when in contact with the L-Cys grafted PCL. The results are displayed in Fig. 5. It is possible to observe that after modification, both materials were antimicrobial, either at 0 h (Fig. 5A) or at $24 \mathrm{~h}$ (Fig. 5B). On immediate contact with grafted foams ( $0 \mathrm{~h}), 1 \mathrm{~h}$ L-Cys-g-PCL (b) showed $97.93 \%$ of significant bacterial reduction, against $77.86 \%$ of $8 \mathrm{~h}$ L-Cysg-PCL (c). For $24 \mathrm{~h}$ the same pattern was observed: $1 \mathrm{~h} \mathrm{L-}$ Cys-g-PCL (b) was significantly higher (99.67\%) than the other (c) of $84.40 \%$.

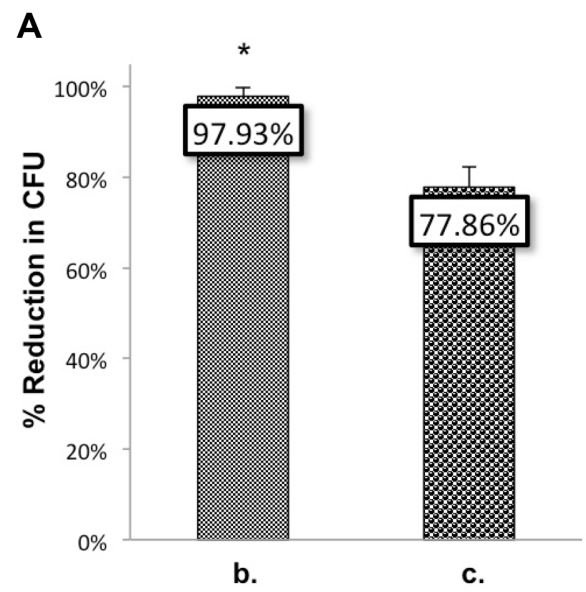

For $1 \mathrm{~h}$ L-Cys-g-PCL, bacteriostatic and bactericidal activity values were calculated, and were 2.70 and 1.38 , respectively. On the other hand, for $8 \mathrm{~h}$ L-Cys-g-PCL, the bacteriostatic and bactericidal activity values were -0.57 and 0.75, respectively. According to JIS L 1902 [30] bacteriostatic and bactericidal activity values for $S$. aureus (ATCC6538P) should be close to 2.3 and 1.5, respectively. The $1 \mathrm{~h}$ L-Cys-g-PCL proved to have bactericidal effect, since its bactericidal value was close to the value of 1.38 .

\section{Scanning Electron Microscopy}

The topography of the cross-sectioned lyophilized foams was analyzed by SEM. Their pore sizes were then measured on SEM images, whose average was obtained using the equation $d=\sqrt{ }(l \times h)$ [31], where $l$ means the maximum pore length and $h$ is the minimum pore length. As illustrated in Fig. 6, PCL foams showed a homogeneous, highly interconnected porous structure. Furthermore, the low TIPS temperature led to low-sized pores and fibers with decreased thickness (thin). The average pore size was $54.65 \pm 10.99 \mu \mathrm{m}$.

There was a significant decrease in the number of adherent $S$. aureus for both conditions of modified samples, after $24 \mathrm{~h}$. For $1 \mathrm{~h}$ L-Cys-g-PCL, almost no bacteria was observed. Regarding $8 \mathrm{~h}$ L-Cys-g-PCL surfaces, they demonstrated, on the other hand, just a small decrease in the number of adherent bacteria when comparing with controls (Fig. 6).

\section{Cytotoxicity Assay}

The $1 \mathrm{~h}$ L-Cys-g-PCL was considered a safe antimicrobial

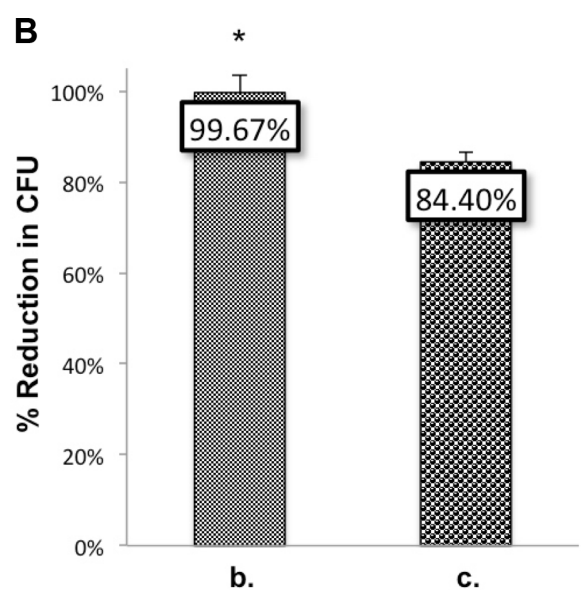

Fig. 5. Percentage of microbial inhibition against $S$. aureus at $0 \mathrm{~h}(\mathbf{A})$ and $24 \mathrm{~h}(\mathbf{B})$.

All of these values were obtained by comparison with results of native polycaprolactone (PCL). Samples tested were $1 \mathrm{~h} \mathrm{L-Cys-g-PCL} \mathrm{(b)} \mathrm{and} 8 \mathrm{~h} \mathrm{~L}-$ Cys-g-PCL (c). * Statistically significant $(p<0.05)$. 
0.
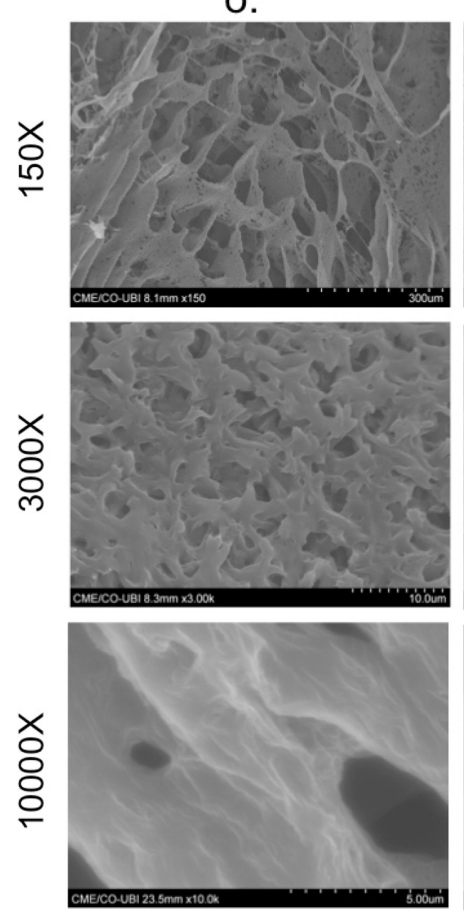

a.
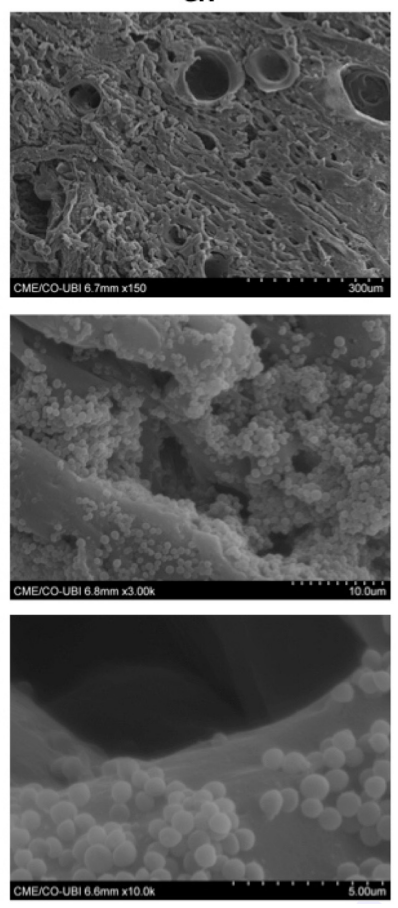

b.
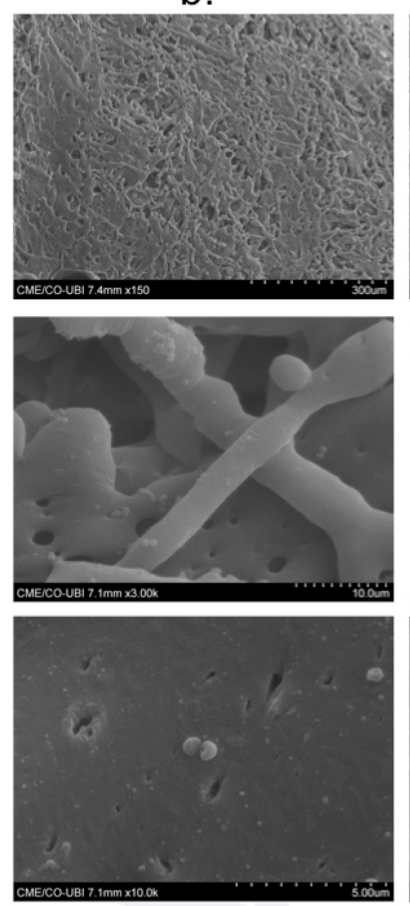

C.
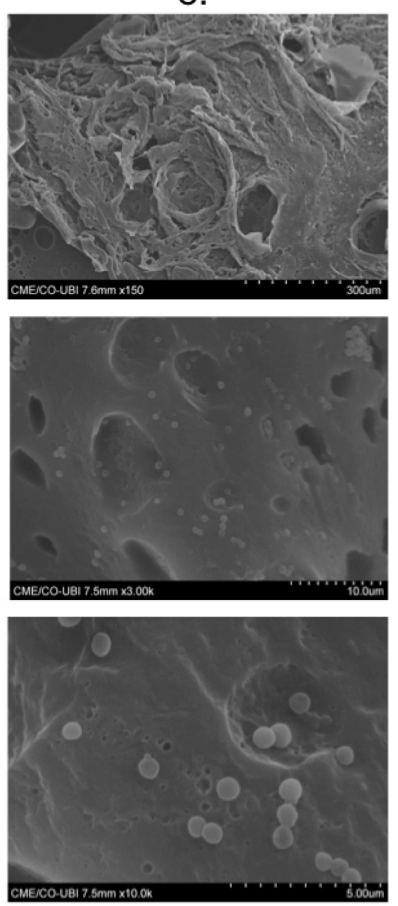

Fig. 6. SEM images of dried polycaprolactone (PCL) foam without bacteria (o).

Adsorbed S. aureus on control PCL (a), $1 \mathrm{~h} \mathrm{L-Cys-g-PCL} \mathrm{(b),} \mathrm{and} 8 \mathrm{~h} \mathrm{L-Cys-g-PCL} \mathrm{(c)} \mathrm{after} 24 \mathrm{~h}$.

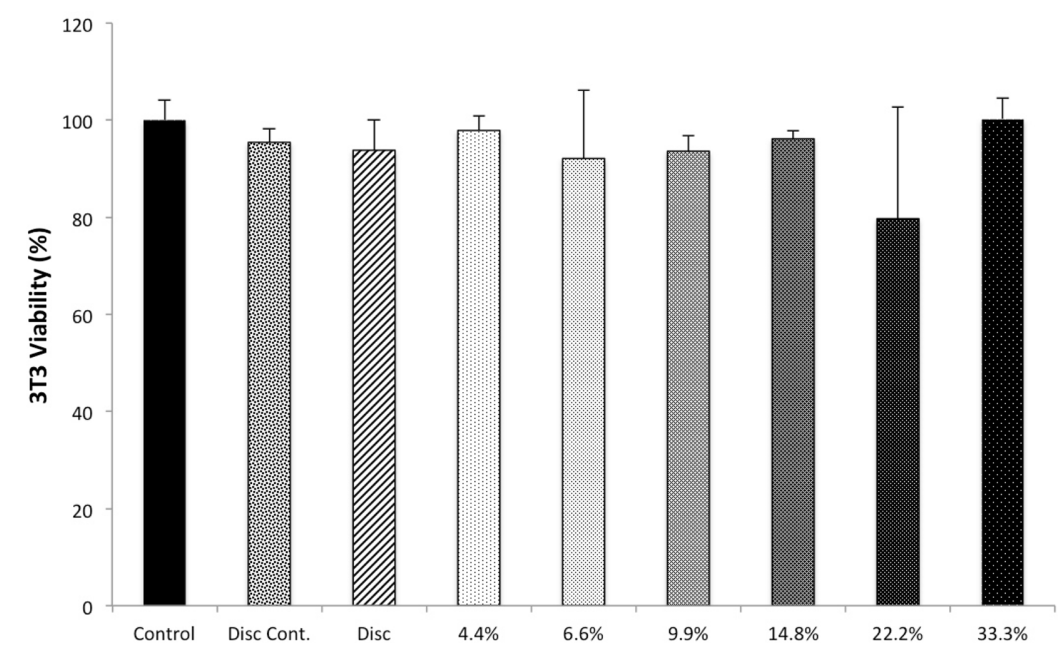

Fig. 7. Mouse fibroblast cell line BALB/c 3 T3 viability percentage when in contact with L-Cys-g-PCL disc functionalized for $1 \mathrm{~h}$ and the perspiration extract of the same condition in different concentrations.

agent on mouse fibroblast cell line BALB/c 3T3 (Fig. 7). Results did not differ beyond $30 \%$ from controls, as shown in Fig. 7. Only a growth inhibition of more than $30 \%$ in comparison with the solvent control is assessed as a clear cell-toxic effect. On the other hand, a growth proliferation of more than $30 \%$ would be considered pro-tumorigenic (E DIN EN ISO 10993-5). In accordance with this, it can be concluded that no cytotoxic substances were released from these samples, avoiding the risk of skin irritation.

\section{Discussion}

This work aims to develop $\mathrm{pH}$-responsive L-Cysteine grafted polyester PCL-based foams for DU treatment. The 
novelties of this work arise with the preparation of L-CysPCL conjugates with high antibacterial activity and the ability to be shaped to fill in sunken holes (craters) - pressure ulcers stage III - acting as a smart material responsive to $\mathrm{pH}$, tailored to develop better swelling properties at alkaline $\mathrm{pH}$ where exudates are normally higher, so as to address exudate self-cleaning and prevention of desiccation. The generated soft materials efficiently inhibited $S$. aureus bacterial growth by the effect of the thiol groups grafted on the polyester.

In the beginning, conservative approaches for healing decubitus/bedsore ulcers recommended their drying and exposure to the environment [5]. In 1962, Winter [34] presented in vivo research, which proved that a humid environment would be more suitable for bedsores healing, whose results were further confirmed in 1963 by Hinman and Maibach [35]. Recent studies have suggested the ideal wound dressing should address exudate self-cleaning, prevention of desiccation, non pro-allergenic effect, gas exchange, protection against dust and toxic contaminants, thermic insulation, and debridement protection $[1,5,36$, $37,38]$. The PCL anionic nature, enhanced by thiolates of LCys [26], renders L-Cys-g-PCL the advantage of preventing electrostatic interactions with negatively charged surfaces, such as mucosa [39], which rely on its negative charge on pendant sulfate groups [40]. This bears debridement protection to the patient. Moreover, an adequate moisture degree elicits a desirable biological medium for cicatrization, allowing a more efficient cellular metabolic activity and activation of growth factors [5].

During this work, the interactions between S. aureus and L-Cys-g-PCL were studied under conditions that simulate DUs; namely, $\mathrm{pH}$ and exudate. The effect of duration of grafting (1 or $8 \mathrm{~h})$ and $\mathrm{pH}$ (7.0 and 8.9$)$ on wettability, surface energy, swelling, and porosity, as well as on S. aureus-L-Cys-g-PCL foam interaction were evaluated.

3D foams were developed from PCL solutions according to the thermally induced phase separation technique, with the posterior sublimation of the solvent. These were subsequently grafted through the covalent reaction aminolysis, with L-Cys. PCL presents ester groups (-COO-) in its structure, which were hydrolyzed to carboxylic acid under slightly acidic condition (solution of $5 \%(\mathrm{w} / \mathrm{v}) \mathrm{L}-\mathrm{Cys} \mathrm{pH}$ 6.5) and heat, through the reverse of Fischer esterification. Amines of L-Cys were covalently grafted on the carboxylic acid of PCL.

The developed application was a porous structure, averaging $54.65 \mu \mathrm{m}$. The porosity controls the amount of fluid of a weeping wound, and it constitutes itself an important parameter for gas exchange. Although the optimal porosity is not fully understood yet, the degree of pore interconnectivity seems more important than its size [21]. The low TIPS temperature led to low-sized, homogeneous, and organized pores and fibers with low thickness. Furthermore, they showed a large surface area and were interconnected.

Readings with EDS, Datacolor, and FT-IR indicated that L-Cys was successfully grafted to PCL foam. Results also demonstrated a higher efficiency of grafting for L-Cys-gPCL foam with $1 \mathrm{~h}$ of functionalization when comparing with $8 \mathrm{~h}$. L-Cys-g-PCL foam increased its volume/swelling after incubation in $\mathrm{pH} 7$ and 8.9. At $\mathrm{pH} 8.9$, the maximum $\mathrm{pH}$ detected in a chronic wound environment [41], the swelling was even more prominent. The mechanism at this alkaline $\mathrm{pH}$ is related to the ionization of hydroxyl and sulfhydryl groups of L-Cys with the consequent repulsion of its chains. L-Cys-g-PCL thus acted as a smart material, responsive to $\mathrm{pH}$. At alkaline $\mathrm{pH} 8.9,1 \mathrm{~h} \mathrm{L-Cys-g-PCL} \mathrm{had}$ swelling ratios of $382 \%$ after $2 \mathrm{~h}$ of incubation in solution, and $431 \%$ after $24 \mathrm{~h}$ of incubation in solution. This is important for a drug-delivery system application, where at physiological $\mathrm{pH}$ chains are tight and compact, whereas at alkaline $\mathrm{pH}$ chains swell and release the drug from inside. Furthermore, depending on the degree of cross-linking with L-Cys, it will affect the crystallinity of the foam structure, and its hydrolysis can lead more or less quickly to the erosion of the wound dressing, with the consequent release of L-Cys. Owing to covalent grafting on a PCL substrate, L-Cys acted as a by-contact antimicrobial, which prevented its leaching to the environment after $24 \mathrm{~h}$ (at $\mathrm{pH}$ 8.9) (data not shown), a condition important to minimize the development of resistance by $S$. aureus.

Surface energies were estimated according to the approach in [42-44]. L-Cys grafting turned PCL from hydrophobic to hydrophilic for both 1 and $8 \mathrm{~h}$ treatments. A change in the surface energy from $7.17 \mathrm{mN} / \mathrm{m}$ for untreated, to 19.14 $\mathrm{mN} / \mathrm{m}$ for $1 \mathrm{~h}$ L-Cys-g-PCL and $19.99 \mathrm{mN} / \mathrm{m}$ for $8 \mathrm{~h} \mathrm{L-Cys-}$ g-PCL was assessed by the boost in the polar part of the surface energy. With extended grafting time $(8 \mathrm{~h})$, although the sample became more hydrophilic than the $1 \mathrm{~h}$ graft, its surface energy remained unaffected. One of the factors that rule water absorbency is the hydrophilicity of the pendant group as well as degree of crosslinking. L-Cys is polar and therefore attracts water, which is also polar. This property made the herein developed foams hydrophilic. One hour of grafting did not show as prominent hydrophilic characteristics as $8 \mathrm{~h}$ of grafting, probably due to the higher porosity, hiding the polar groups. Moreover, for $1 \mathrm{~h} \mathrm{L-Cys-}$ 
g-PCL, the higher XRD peak relative intensity, as shown in Fig. 2, was evidence that suggested a higher crystalline structure for this material. Melting temperatures $\left(T_{\mathrm{m}}\right)$ also decreased (Fig. 3), which implied that aminolysis was successfully achieved. Furthermore, L-Cys covalently bonded to ester made it difficult to break, which left weaker intermolecular forces easier to break. Moreover, its decreased enthalpy of fusion, $0.58 \mathrm{~mW} / \mathrm{mg}$, explains the chain breakage in two parts.

In accordance with the chemical surface analyses for $1 \mathrm{~h}$ L-Cys-g-PCL, microbiologic data for $S$. aureus at 0 and $24 \mathrm{~h}$ of incubation showed $97.93 \%$ and $99.67 \%$, respectively. Such an inhibition is sustainable because L-Cys, when adsorbed to the foam, has the thiol groups more readily available to react with bacterial proteins and cause a bactericidal effect [26], compromising the membrane metabolic and bioenergetic machinery [27]. On the other hand, for $8 \mathrm{~h}$ L-Cys-g-PCL, the killing rates decreased for $77.86 \%$ and $84.40 \%$ for 0 and $24 \mathrm{~h}$, respectively. This is understandable by the less efficient grafting of L-Cys. The shape and amount of S.aureus were evaluated, under electron microscopy, when adsorbed on $1 \mathrm{~h}$ L-Cys-g-PCL and $8 \mathrm{~h} \mathrm{L-Cys-g-PCL}$ for $24 \mathrm{~h}$. It was demonstrated that fewer bacteria adhered to grafted foams when comparing with controls, albeit $1 \mathrm{~h} \mathrm{L-Cys-g-PCL}$ had the lowest and debilitated adhered S. aureus.

These interactions between S. aureus and L-Cys-g-PCL are partly in accordance with the theoretical model predicted, in that albeit the unfavorable capacity of $S$. aureus to adsorb the native PCL foam $\left(\Delta G_{\text {Adhesion }}{ }_{\text {TOT }}=7.17\right)$, when they were exposed to the modified foam, namely $1 \mathrm{~h}$ L-Cys-g-PCL $\left(\Delta G_{\text {Adhesion }}{ }^{\text {TOT }}=19.14\right)$ and $8 \mathrm{~h} \mathrm{L-Cys-g-PCL}\left(\Delta G_{\text {Adhesion }}{ }_{\text {TOT }}=\right.$ $19.99)$, as the $\Delta G_{\text {Adhesion }}{ }^{\text {TOT }}$ increased to similar values, the adhesion capacity of S.aureus to grafted PCL would decrease equally for both. Different porosity between $1 \mathrm{~h} \mathrm{~L}$ Cys-g-PCL and $8 \mathrm{~h}$ L-Cys-g-PCL may explain this fact. Furthermore, bacterial phase variation mechanisms, which fluctuate with $\mathrm{pH}$, may have influence on the $S$. aureus wall. It can express different extracellular structures, such as fimbriae and extracellular polymeric substances [45], which can protect $S$. aureus better from inefficient grafting or lower porosity at $8 \mathrm{~h} \mathrm{~L}$-Cys-g-PCL.

After comparing with the literature [1, 5, 41, 46-50], the herein developed wound dressing is foam-shaped and composed by a polymeric material, with absorbence eased by the incorporated pores, and with potential to be semiocclusive. This absorption capacity varied according to foam porosity, as well as grafted L-Cys. It can be used to drain wounds, and to work as a pressure relief wound dressing. Moreover, it is gas and water vapor permeable (porosity), and offers protection against shear forces. This novel material is proposed to be in contact with the wound surface, absorbing its exudate, which can contribute to the moisture environment, once its safety is proved against 3T3 fibroblasts. Although it is stated that foam wound dressings should not be used in infected wounds [5,50], this research work shows other perspective.

This study allowed the covalent hydrophobic-tohydrophilic conversion of a PCL substrate via aminolysis with L-Cys. This was demonstrated by the contact angle, free energy of adhesion, X-ray spectroscopy, infrared spectroscopy, X-ray diffraction analysis, differential scanning calorimetry, and scanning electron microscopy.

L-Cys showed optimal grafting to PCL foam with $1 \mathrm{~h}$ of functionalization under slight acidic environment and heat. In accordance with the chemical data, microbiologic results for S. aureus at $0 \mathrm{~h}$ and $24 \mathrm{~h}$ of incubation showed $97.93 \%$ and $99.67 \%$ of bacterial reduction, respectively, which revealed our application to be microbicidal. Furthermore, the number of bacteria adhered after $24 \mathrm{~h}$ was negligible and with a compromised morphology.

A new pH-responsive smart material with antioxidant, safety, and swelling properties, ability to be shaped to fill in sunken holes (pressure ulcers stage III), and strong antimicrobial properties is herein presented for potential use as a wound dressing for decubitus ulcers.

\section{Acknowledgments}

The authors acknowledge the Fundação para a Ciência e Tecnologia (FCT) for funding the PhD grant SFRH/BD/ 91444/2012 and Programa Operacional Capital Humano $(\mathrm{POCH})$ and European Union for co-funding it.

\section{References}

1. Fonder MA, Lazarus GS, Cowan DA, Aronson-Cook B, Kohli AR, Mamelak AJ. 2008. Treating the chronic wound: a practical approach to the care of nonhealing wounds and wound care dressings. J. Am. Acad. Dermatol. 58: 185-206.

2. Bansal C, Scott R, Stewart D, Cockerell CJ. 2005. Decubitus ulcers: a review of the literature. Int. J. Dermatol. 44: 805-810.

3. Thomas DR. 2001. Improving outcome of pressure ulcers with nutritional interventions: a review of the evidence. Nutrition 17: 121-125.

4. Sen CK, Gordillo GM, Roy S, et al. 2009. Human skin wounds: a major and snowballing threat to public health and the economy. Wound Repair Regen. 17: 763-771.

5. Shai A, Maibach HI. 2005. Wound Healing and Ulcers of the 
Skin: Diagnosis and Therapy - The Practical Approach. Springer, New York. USA.

6. Biswas S, Roy S, Banerjee J, Hussain SRA, Khanna S, Meenakshisundaram G, et al. 2010. Hypoxia inducible microRNA 210 attenuates keratinocyte proliferation and impairs closure in a murine model of ischemic wounds. Proc. Natl. Acad. Sci. USA 107: 6976-6981.

7. Khioka S, Ando T, Shibata M, Sekiya N, Nakatsuka T. 2008. Oxygen consumption of keloids and hypertrophic scars. Ann. Plastic Surg. 60: 194-197.

8. Osinsky S, Zavelevich M, Vaupel P. 2009. Tumor hypoxia and malignant progression. Exp. Oncol. 31: 80-86.

9. Bauer SM, Bauer RJ, Velazquez OC. 2005. Angiogenesis, vasculogenesis, and induction of healing in chronic wounds. Vasc. Endovascular Surg. 39: 293-306.

10. Baker EA, Leaper DJ. 2000. Proteinases, their inhibitors, and cytokine profiles in acute wound fluid. Wound Repair Regen. 8: 392-398.

11. Trengove NJ, Bielefeldt-Ohmann H, Stacey MC. 2000. Mitogenic activity and cytokine levels in non-healing and healing chronic leg ulcers. Wound Repair Regen. 8: 13-25.

12. Gautam N, Olofsson AM, Herwald H, Iversen LF, LundgrenAkerlund E, Hedqvist P, et al. 2001. Heparin-binding protein (HBP/CAP37): a missing link in neutrophil-evoked alteration of vascular permeability. Nat. Med. 7: 1123-1127.

13. Lundqvist K, Herwald H, Sonesson A, Schmidtchen A. 2004. Heparin binding protein is increased in chronic leg ulcer fluid and released from granulocytes by secreted products of Pseudomonas aeruginosa. Thromb. Haemost. 92: 281-287.

14. Gray M, Weir D. 2007. Prevention and treatment of moisture-associated skin damage (maceration) in the periwound skin. J. Wound Ostomy Continence Nurs. 34: 153-157.

15. Halcon L, Milkus K. 2004. Staphylococcus aureus and wounds: a review of tea tree oil as a promising antimicrobial. Am. J. Infect. Control 32: 402-408.

16. McGuiness W, Vella E, Harrison D. 2004. Influence of dressing changes on wound temperature. J. Wound Care 13: 383-385.

17. Mathus-Vliegen EM. 2004. Old age, malnutrition, and pressure sores: an ill-fated alliance. J. Gerontol. A Biol. Sci. Med. Sci. 59: 355-360.

18. Freiman A, Bird G, Metelitsa AI, Barankin B, Lauzon GJ. 2004. Cutaneous effects of smoking. J. Cutan. Med. Surg. 8: 415-423.

19. Loncarevic S, Jørgensen HJ, Løvseth A, Mathisen T, Rørvik LM. 2005. Diversity of Staphylococcus aureus enterotoxin types within single samples of raw milk and raw milk products. J. Appl. Microbiol. 98: 344-350.

20. Chambers HF, DeLeo FR. 2009. Waves of resistance: Staphylococcus aureus in the antibiotic era. Nat. Rev. Microbiol. 7: 629-641.

21. Buck DW, Goucher H, Lewis VL. 2012. The incidence of methicillin-resistant Staphylococcus aureus in pressure ulcers.
Adv. Skin Wound Care 25: 509-512.

22. Ratner BD. 2004. Biomaterials Science: An Introduction to Materials in Medicine. 2nd Ed. Elsevier Academic Press, Amsterdam. Netherlands.

23. Ikada Y, Tsuji H. 2000. Biodegradable polyesters for medical and ecological applications. Macromol. Rapid Commun. 21: 117-132.

24. Ulery BD, Nair LS, Laurencin CT. 2011. Biomedical applications of biodegradable polymers. J. Polym. Sci. B Polym. Phys. 49: 832-864.

25. Gross RA, Kalra B. 2000. Biodegradable polymers for the environment. Science 297: 803-807.

26. Nogueira F, Gonçalves IC, Martins MCL. 2013. Effect of gastric environment on Helicobacter pylori adhesion to a mucoadhesive polymer. Acta Biomater. 9: 5208-5215.

27. Caldeira E, Piskin E, Granadeiro L, Silva F, Gouveia IC. 2013. Biofunctionalization of cellulosic fibers with L-cysteine: assessment of antibacterial properties and mechanism of action against $S$. aureus and K. pneumoniae. J. Biotechnol. 168: 426-435.

28. Haslekås C, Viken MK, Grini PE, Nygaard V, Nordgard SH, Meza TJ, Aalen RB. 2003. Seed 1-cysteine peroxiredoxin antioxidants are not involved in dormancy, but contribute to inhibition of germination during stress. Plant Physiol. 133: 1148-1157.

29. Bayoudh S, Ponsonmet L, Ouada HB, Bakhrouf A, Othmane A. 2005. Bacterial detachment from hydrophilic and hydrophobic surfaces using a microjet impingement. Colloid Surf. A Physicochem. Eng. Asp. 266: 160-167.

30. Japanese Standards Association. 2012. Japanese Industrial Standard L 1902: Testing for antibacterial activity and efficacy on textile products. JSA, Japan.

31. Amaral IF, Sampaio P, Barbosa MA. 2006. Threedimensional culture of human osteoblastic cells in chitosan sponges: the effect of the degree of acetylation. J. Biomed. Mater. Res. A 76: 335-46.

32. Socrates G. 2004. Infrared and Raman Characteristic Group Frequencies: Tables and Charts. John Wiley \& Sons, Ltd, New York. USA.

33. $\mathrm{Hu} \mathrm{H}$, Dorset DL. 1990. Crystal structure of poly(iecaprolactone). Macromolecules 23: 4604-4607.

34. Winter GD. 1962. Formation of the scab and the rate of epithelization of superficial wounds in the skin of the young domestic pig. Nature 193: 293-294.

35. Hinman CD, Maibach H. 1963. Effect of air exposure and occlusion on experimental human skin wounds. Nature 200: 377-378.

36. Morin RJ, Tomaselli NL. 2007. Interactive dressings and topical agents. Clin. Plast. Surg. 34: 643-658.

37. Nemeth AJ, Eaglstein WH, Taylor JR, Peerson LJ, Falanga V. 1991. Faster healing and less pain in skin biopsy sites treated with an occlusive dressing. Arch. Dermatol. 127: 16791683. 
38. Seaman S. 2002. Dressing selection in chronic wound management. J. Am. Podiatr. Med. Assoc. 92: 24-33.

39. Smart JD, Kellaway IW, Worthington HE. 1984. An in vitro investigation of mucosa-adhesive materials for use in controlled drug delivery. J. Pharm. Pharmacol. 36: 295-299.

40. Capra RH, Baruzzi AM, Quinzani LM, Strumia MC. 2007. Rheological, dielectric and diffusion analysis of mucin/ carbopol matrices used in amperometric biosensors. Sens. Actuators B Chem. 124: 466-476.

41. Gethin G. 2007. The significance of surface $\mathrm{pH}$ in chronic wounds. Wounds UK 3: 52-56.

42. Van Oss CJ, Chaudhury MK, Good RJ. 1987. Monopolar surfaces. Adv. Colloid Interface Sci. 28: 35-64.

43. Van Oss CJ, Good RJ, Chaudhury MK. 1988. Additive and nonadditive surface tension components and the interpretation of contact angles. Langmuir 4: 884-891.

44. Van Oss CJ, Ju L, Chaudhury MK, Good RJ. 1989. Estimation of the polar parameters of the surface tension of liquids by contact angle measurements on gels. J. Colloid Interface Sci. 128: 313-319.

45. Nogueira F, Mouro C, Piskin E, Gouveia I. 2014. Covalent modification of cellulosic-based textiles: a new strategy to obtain antimicrobial properties. Biotechnol. Bioprocess Eng. 19: 526-533.

46. Eaglstein WH, Mertz PM, Falanga V. 1987. Occlusive dressings. Am. Fam. Physician 35: 211-216.

47. Fletcher J. 2005. Understanding wound dressings: foam dressings. Nurs. Times 101: 50-51.

48. Falanga V. 2001. Cutaneous Wound Healing, 1st Ed. Martin Dunitz, London. UK.

49. Taylor BA. 2003. Selecting wound healing products. Choices for long-term care settings. Adv. Nurse Pract. 11: 63-66.

50. Ohura N, Ichioka S, Nakatsuka T, Shibata M. 2005. Evaluating dressing materials for the prevention of shear force in the treatment of pressure ulcers. J. Wound Care 14: 401-404. 